\title{
Temperature and Doping Dependencies of Electron Mobility in InAs, AlAs and AlGaAs at High Electric Field Application
}

\author{
H. Arabshahi ${ }^{1}$, M. R. Khalvati ${ }^{2}$, and M. Rezaee Rokn-Abadi ${ }^{1}$ \\ ${ }^{1}$ Department of Physics, Ferdowsi University of Mashhad, Mashhad, Iran \\ ${ }^{2}$ Tarbiat Moallem University of Sabzevar, \\ P.O.Box 397, Sabzevar, Iran, Email: hadi.arabshahi@sttu.ac.ir
}

(Received on 27 January, 2008)

\begin{abstract}
Temperature and doping dependencies of electron mobility in InAs, AlAs and AlGaAs structures have been calculated using an ensemble Monte Carlo simulation. Electronic states within the conduction band valleys at the $\Gamma, L$ and $X$ are represented by non-parabolic ellipsoidal valleys centred on important symmetry points of the Brillouin zone. The simulation shows that intervalley electron transfer plays a dominant role in high electric fields leading to a strongly inverted electron distribution and to a large negative differential conductance. Our simulation results have also shown that the electron velocity in InAs and AlAs is less sensitive to temperature than in other III-V semiconductors like GaAs and AlGaAs. So InAs and AlAs devices are expected be more tolerant to self-heating and high ambient temperature device modeling. Our steady-state velocity-field characteristics are in fair agreement with other recent calculations.
\end{abstract}

Keywords: Electron Mobility; Steady-state; Ellipsoidal valleys; Self-heating

\section{INTRODUCTION}

GaAs and related compounds with aluminium and indium currently have great potential for applications in optoelectronic devices, high frequency applications and quantum dots (QD's) infrared photodetectors [1-4]. Different GaAs/AlAs electronic and optoelectronic devices with quantum effects are currently commercially available. An increasing tendency in such devices is the implementation of complicated multilayered nano-systems, containing two or more components interacting with each other. The Monte Carlo technique has proved valuable for studying non-equilibrium carrier transport in a range of semiconductor materials and devices [5-8], and electron transport in bulk semiconductor was modelled as long ago as 1966 [9]. However, carrier transport modelling has only recently begun to receive sustained attention [10-11], now that the growth of compounds and alloys is able to produce viable material for the electronics industry. As the start of a programme of work modelling high field transport and breakdown in InAs, AlAs and AlGaAs, this paper presents the results of Monte Carlo simulations of electron transport in bulk InAs, AlAS and AlGaAs at different temperature and electron concentrations. The simulations have been carried out using a non-parabolic ellipsoidal valley model to describe transport in the conduction band. This article is organised as follows.

Details of the conduction band parameters and the Monte Carlo simulation are presented in section 2, and the results of steady state and transient transport simulations are discussed in section 3 .

\section{SIMULATION MODEL}

In order to calculate the electron drift velocity for large electric fields, consideration of conduction band satellite val- leys is necesasry. The first-principles band structure of zincblende InAs, AlAs and AlGaAs predicts a direct band gap located at the $\Gamma$ point and lowest energy conduction band satellite valleys at the $X$ point and at the $L$ point. In our Monte Carlo simulation, the $\Gamma$ valley, the three equivalent $X$ valleys, the four equivalent $L$ valleys, are represented by ellipsoidal, nonparabolic dispersion relationships of the following form [12-13]

$$
E(\mathbf{k})\left[1+\alpha_{i} E(\mathbf{k})\right]=\frac{\hbar^{2} k^{2}}{2 m^{*}}
$$

where $m^{*}$ is effective mass at the band edge and $\alpha_{i}$ is the nonparabolicity coefficient of the $i$-th valley. The band structure and material parameters necessary for calculating the scattering probabilities used in the present Monte Carlo simulation are given in table 1 and 2. Scattering mechanisms included in the simulation are acoustic deformation potential scattering (treated either as an elastic process or as an inelastic process) and pizoelectric scattering (which is found to be negligible in the temperature range discussed here). Furthermore, longitudinal optical phonon scattering, nonequivalent and, where applicable, equivalent intervalley scattering events are taken into account among all valley types with the transfers assumed to be governed by the same deformation potential fields and the same phonon frequencies. Degeneracy effects are expected to be negligible over almost all of the temperature and electron concentration ranges of interest here and, hence, are not considered in the calculation.

In our model at the start of each simulation, ten thousand electron particles are distributed in momentum space according to a Maxwell-Boltzmann distribution function. These particles are propagated classically between collisions according to thier velocity, effective mass and the prevailing field. The selection of the propagation time, scattering mechanism and other related quantities is achieved by generating random numbers and using this numbers to select, for example, a scattering mechanism. In the case of the ellipsoidal, non-parabolic 
conduction valley model, the usual Herring-Vogt transformation matrices are used to map carrier momenta into spherical valleys when particles are drifted or scattered. Steady-state results of high field transport studies have been obtained for lattice temperatures up to $400 \mathrm{~K}$, in order to gain some insight into the hot carrier transport and the energy distribution function that would be generated in the gate-drain region of a power field effect transistor.

TABLE I: Important band parameters used in the Monte Carlo simulation for zincblende AlAs, AlGaAs and InAs.

\begin{tabular}{l|cccc}
\hline \hline & Valley & $E_{\text {gap }}(\mathrm{eV})$ & $m^{*}$ & Nonparabolicity $\left(\mathrm{eV}^{-1}\right)$ \\
\hline AlAs & $\Gamma$ & 2.95 & 0.146 & 0.25 \\
& $\mathrm{~L}$ & 3.05 & 0.19 & 0.029 \\
& $\mathrm{X}$ & 3.2 & 0.6 & 0.08 \\
\hline AlGaAs & $\Gamma$ & 1.82 & 0.086 & 0.55 \\
& $\mathrm{~L}$ & 1.93 & 0.233 & 0.62 \\
& $\mathrm{X}$ & 1.97 & 0.411 & 0.325 \\
\hline InAs & $\Gamma$ & 0.356 & 0.027 & 2.66 \\
& $\mathrm{~L}$ & 0.73 & 0.64 & 0.7 \\
& $\mathrm{X}$ & 1.02 & 0.286 & 1.5 \\
\hline \hline
\end{tabular}

TABLE II: Material parameter selections for AlAs, AlGaAs, and InAs.

\begin{tabular}{l|ccc}
\hline \hline & AlAs & AlGaAs & InAs \\
\hline Density $\rho\left(\mathrm{kgm}^{-3}\right)$ & 5667 & 4200 & 5370 \\
Longitudinal sound velocity $v_{s}\left(\mathrm{~ms}^{-1}\right)$ & 4280 & 5130 & 5500 \\
Low-frequency dielectric constant $\varepsilon_{s}$ & 14.6 & 11.4 & 12.5 \\
High-frequency dielectric constant $\varepsilon_{\infty}$ & 12.25 & 10.5 & 8.7 \\
Acoustic deformation potential $\mathrm{D}(\mathrm{eV})$ & 4.9 & 8.3 & 8.8 \\
Polar optical phonon energy $\hbar \omega_{p o}(\mathrm{eV})$ & 0.015 & 0.09 & 0.02 \\
Energy gap $(\mathrm{eV})$ & 0.356 & 1.82 & 1.43 \\
Lattice constant $a_{0}(\AA)$ & 3.39 & 2 & 6.2 \\
Intervalley deformation potentials $\left(10^{7} \mathrm{eVm}^{-1}\right)$ & 1 & 1 & 1 \\
Intervalley phonon energies $(\mathrm{meV})$ & 11.2 & 26 & 32.2 \\
\hline \hline
\end{tabular}

\section{RESULTS}

Electron drift velocity as a function of electric field is important in determining the performance of high-speed and microwave semiconductor devices. Here we show the results of temperature dependence of the steady-state velocity-field characteristics and valley occupancy in bulk InAs, AlAs and AlGaAs materials. Fig. 1 shows the simulated velocity-field characteristics at $300 \mathrm{~K}$, with a background doping concentration of $10^{17} \mathrm{~cm}^{-3}$, and with the electric field applied along one

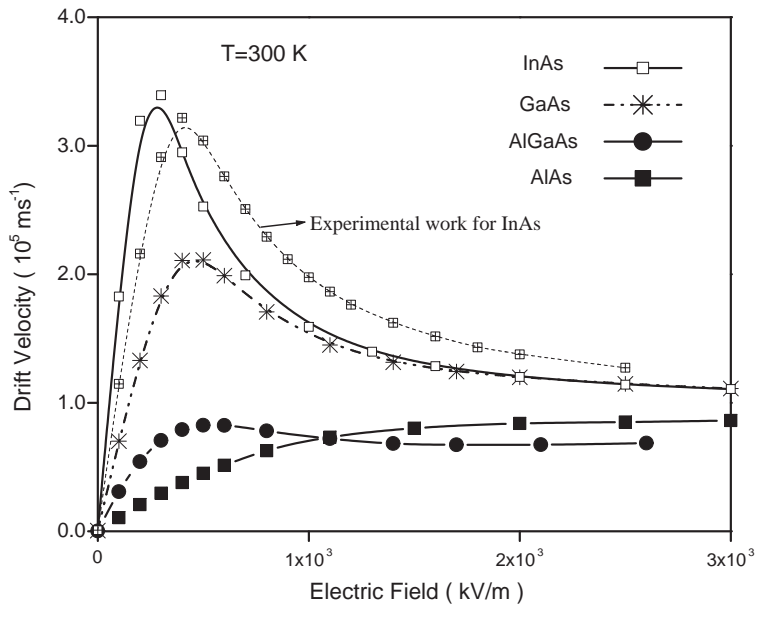

FIG. 1: Calculated steady-state electron drift velocity in bulk zincblende InAs, AlAs and AlGaAs using non-parabolic band models in comparison with GaAs material and experimental measurments of Brennan et al. [14].

of the cubic axes.

Similar to the experimental results the simulations suggest that the peak drift velocity for zincblende InAs is $\sim 3.4 \times 10^{5}$ $\mathrm{ms}^{-1}$ while those for AlAs and AlGaAs are about $0.8 \times 10^{5}$ $\mathrm{ms}^{-1}$ and $10^{5} \mathrm{~ms}^{-1}$, respectively. At higher electric fields, intervalley optical phonon emission dominates, causing the drift velocity to saturate at around $1.2 \times 10^{5} \mathrm{~ms}^{-1}$. The calculated high field electron drift velocity apparent from Fig. 1 is fractionally lower than those that have been simulated by Adachi et al. [4-5] who assumed an effective mass in the upper valleys equal to the free electron mass. The threshold field for the onset of significant scattering into satellite conduction band valleys is a function of the intervalley separation and the density of electronic states in the satellite valleys.

The importance of electron intervalley transfer at high electric fields can be clearly seen in Fig. 2. In this figure the fractional valley occupancies for different materials is plotted. It is obvious that the inclusion of satellite valleys in the simulations is important. Significant electron transfer to the upper valleys only begins to occur when the field strength is very close to the threshold value. At the threshold field the electron valley occupancies at room temperature for $\Gamma, L$ and $X$ are about $85 \%, 13 \%$ and $2 \%$, respectively.

Figure 3 shows the calculated electron drift velocity as a function of electric field strength for temperatures of 300 , 350 and $400 \mathrm{~K}$. The decrease in drift mobility with temperature at low fields is due to increased intravalley polar optical phonon scattering whereas the decrease in velocity at higher fields is due to increased intra and intervalley scattering. It can be seen from the figure that the peak velocity also decreases and moves to higher electric field as the temperature is increased. This is due to the general increase of total scattering rate with temperature, which suppresses the electron energy and reduces the population of the satellite valleys. This latter effect is apparent from the fact that the electron population in the central $\Gamma$-valley increases with temperature as shown in 

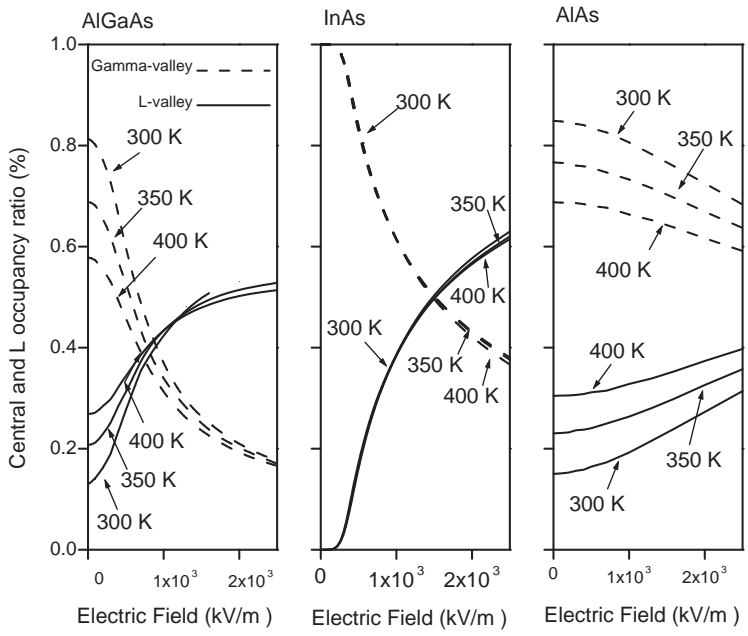

FIG. 2: Fractional occupation of the central and satellite valleys in InAs, AlAs and AlGaAs as a function of applied electric field using the non-parabolic band model at different temperatures up to $400 \mathrm{~K}$.

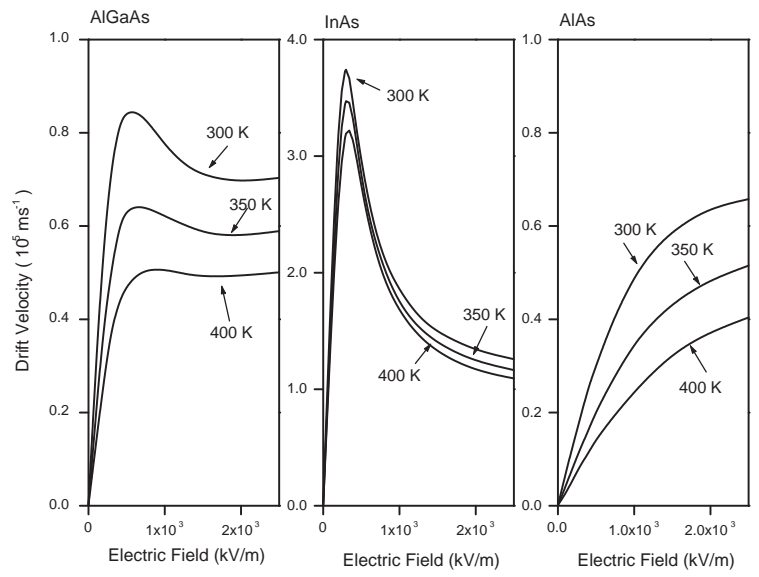

FIG. 3: Calculated electron steady-state drift velocity in bulk InAs, $\mathrm{AlAs}$ and $\mathrm{AlGaAs}$ as a function of applied electric field at various lattice temperatures and assuming a donor concentration of $10^{17} \mathrm{~cm}^{-3}$. The peak drift velocity decreases while the threshold field increases by same percent as the lattice temperature increases from 300 to 400 $\mathrm{K}$.

Fig. 2.

Figure 4 shows how the velocity-field relation changes with temperature for electrons in the most populated $\Gamma$ and $L$ valleys. There are significant statistical fluctuations in the results for the drift velocity of electrons in the $L$ valleys for fields around $500 \mathrm{kVm}^{-1}$, which are caused by the relatively small number of electron particles occupying the valleys just above the threshold for intervalley transfer. Nevertheless it can be

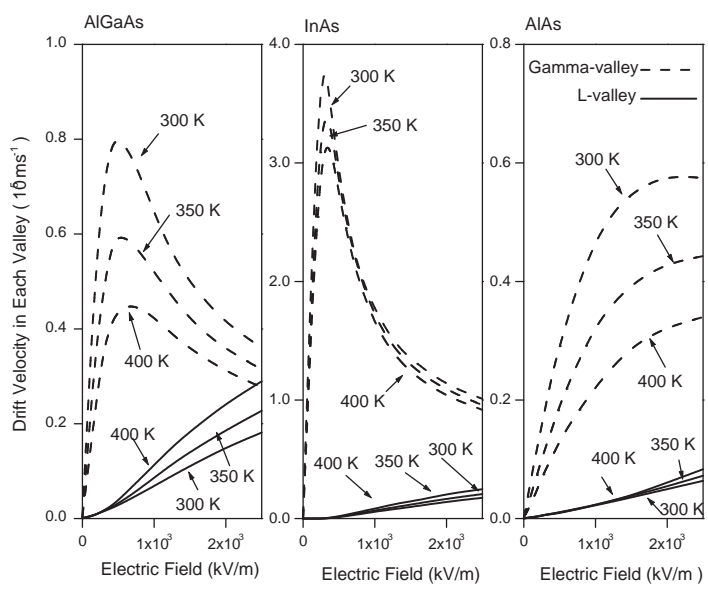

FIG. 4: Temperature dependence of the drift velocity in the $\Gamma$ and $L$ valleys of InAs, AlAs and AlGaAs materials as a function of applied electric field.

seen that the average drift velocity decreases as the temperature increases for both valleys. Comparison of the temperature dependence of the transport properties in InAs, AlAs and AlGaAs materials show that the change in peak velocity of AlGaAs from $300 \mathrm{~K}$ to $400 \mathrm{~K}$ is a reduction of about $45 \%$ whereas for InAs and AlAs, it is about $25 \%$ and $20 \%$, respectively. The reason can be explained in terms of the energy band structure. In particular, the different electron effective mass within the central valley $\left(0.027 \mathrm{~m}_{0}\right.$ in InAs versus 0.086 $\mathrm{m}_{0}$ and $0.146 \mathrm{~m}_{0}$ in AlGaAs and AlAs, respectively). This is important because electrons which are near a valley minimum have small kinetic energies and are therefore strongly scattered. It is apparent that intervalley transfer is substantially larger in InAs over the range of applied electric fields shown, due to the combined effect of a lower $\Gamma$ effective mass, lower satellite valley separation energy, and slightly lower phonon scattering rate within the $\Gamma$ valley.

Therefore, the electron velocity in InAs and AlAs is less sensitive to temperature than in AlGaAs, so InAs devices are expected be more tolerant to self-heating and high ambient temperature.

Figure 5 shows how the velocity-field characteristic of InAs, $\mathrm{AlAs}$ and $\mathrm{AlGaAs}$ materials change with impurity concentration at $300 \mathrm{~K}$. It is clear that with increasing donor concentration, there are reduction in the average peak drift velocity and the threshold field because of increasing scattering rate events. The results show the trend expected from increased ionized impurity scattering is in good general agreement with recent calculations by other workers [14-16].

\section{CONCLUSIONS}

Electron transport at different temperatures in bulk zincblende InAs, AlAs and $\mathrm{AlGaAs}$ have been simulated 


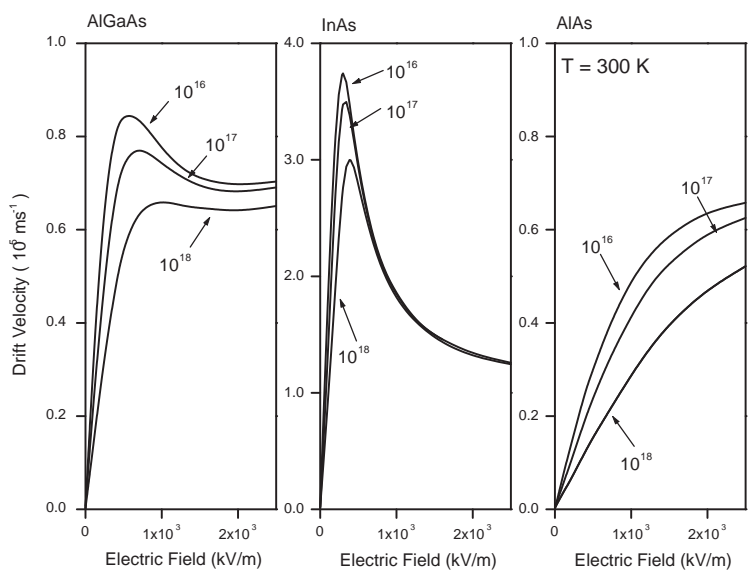

FIG. 5: Calculated electron steady-state drift velocity in bulk InAs, AlAs and AlGaAs as a function of applied electric field at various donor concentration up to $10^{18} \mathrm{~cm}^{-3}$ at room temperature. The peak drift velocity decreases due to increasing scattering rate. using an ensemble Monte Carlo simulation. Using valley models to describe the electronic bandstructure, calculated velocity-field characteristics show that the intervalley transitions in high electric fields play an important role in these materials. The intervalley transitions lead to a large negative differential conductance. Saturation drift velocities of about $1.2 \times 10^{5} \mathrm{~ms}^{-1}$ match recent measurements on low-doped bulk samples. We have also demonstrated that low temperature sensitivity of the electron transport properties of InAs and AlAs is attractive for high-temperature and high-power electronic applications.

\section{ACKNOWLEDGMENTS}

This work is be benefited from useful discussion with $\mathrm{M}$. G. paeezi.
[1] H. Cheong, Y. J. Jeon, and H. Hwang,. J. Korean Physical Scciety., 44, 697 (2004).

[2] J. Maes, M. Hayne, Y. Sider, and B. Partoens, Physical Review B., 70, 155311 (2004)

[3] A. H. You, D. S. Ong, Proc. ICSE 2000., 182 (2000).

[5] S. Adachi, GaAs and Related Materials, Bulk semiconducting and Superlattice Properties, World Scientific, Singapore, 1994.

[5] S. Adachi, Physical properties of III-V Semiconductor Compounds, InP, InAs, GaAs, GaP,AlAs and AlGaAs Wiley, New York, 1992.

[6] M. Allali, C. B. Sorensen, E. Veje, and P. Tidemand-Petersson, Phys. Rev. B 48, 4398 (1993).

[7] S. J. Lee, L. Y. Juravel, J. C. Wooley, and A. J. Spring, Phys. Rev. B 21, 659 (1980).

[8] B. E. Foutz, L. F. Eastman, U. V. Bhapkar, and M. Shur, Appl. Phys. Lett. 70, 2849 (1997).
[9] T. Kurosawa, J. Phys. Soc. Jpn., 21, 424 (1966).

[10] C. Moglestue, Monte Carlo Simulation of Semiconductor Devices 1993 pub. Chapman and Hall.

[11] C. Jacoboni and P. Lugli, The Monte Carlo Method for semiconductor and Device Simulation 1989 pub. Springer-Verlag.

[13] H. Arabshahi, M. R. Benam, and B. Salahi Modern Physics Letters B. 21, 1715 (2007).

[13] H. Arabshahi, Modern Physics Letters B. 21, 199 (2007).

[14] K. Brennan, K. Hess, J. Y. Tang, and G. J. Iafrate, IEEE Trans. Electron Devices, 30, 1750 (1983).

[16] N. Newman, T. Kendelewicz, L. Bowman, and W. E. Spicer, Appl. Phys. Lett. 46, 1176 (1985).

[16] N. Newman, V. Schilfgaarde, T. Kendelewicz, and W. E. Spicer, Mater. Res. Soc. Symp. Proc. 54, 443 (1986). 